\begin{tabular}{lc}
\hline & Sharif University of Technology \\
SCIENTIA & Transactions B: Mechanical Engineering \\
IRAN ICA & www.scientiairanica.com \\
\hline
\end{tabular}

\title{
A parametric study of optimal number and location of radiant heaters in enclosures with participating media
}

\author{
H. Amiri ${ }^{a, *}$ and P.J. Coelho ${ }^{b}$ \\ a. Department of Energy, Institute of Science and High Technology and Environmental Sciences, Graduate University of Advance \\ Technology, Haftbagh Highway, Kerman, P.O. Box 76315-117, Iran. \\ b. IDMEC, LAETA, Instituto Superior Técnico, Universidade de Lisboa Av. Rovisco Pais 1, 1049-001 Lisboa, Portugal.
}

Received 24 April 2014; received in revised form 3 February 2015; accepted 13 April 2015

\author{
KEYWORDS \\ Optimization; \\ Participating media; \\ Micro-genetic \\ algorithm; \\ Discrete ordinates \\ method; \\ Anisotropic \\ scattering.
}

\begin{abstract}
An inverse solution technique is applied to the design of radiant enclosures when design variables are discrete and radiation is the dominant mode of heat transfer. The enclosure contains an absorbing, emitting and linear anisotropic scattering medium in radiative equilibrium. The discrete ordinate method is employed to solve the radiative transfer equation. The goal of the design problem is to find the best number and location of discrete equally powered heaters which produce the desired (specified) temperature and heat flux profile over the design surface of enclosures. The inverse problem is formulated as an optimization problem and is solved using a micro-genetic algorithm. Results show that the micro genetic algorithm is able to find the optimal solution by just searching a few percent of feasible solutions. The ability of this methodology is demonstrated by finding the optimal number and location of heaters in an irregular enclosure. Then, the effect of some thermophysical properties, such as extinction coefficient, scattering albedo, scattering phase function, and design surface emissivity, on the optimal solution is considered.

(C) 2016 Sharif University of Technology. All rights reserved.
\end{abstract}

\section{Introduction}

High-temperature furnaces and ovens are used extensively in industry to provide energy to engineering processes. In these systems, radiative heat transfer is important and often is the dominant mode of heat transfer. These systems typically consist of an array of heaters/burners that are placed on the top, bottom, and sometimes the sides of the enclosure and which provide a supply of thermal radiation to the load inside them. In the design of such systems, the design goal is to find the number and/or location and/or power of the heaters/burners that produce the desired pre-specified

*. Corresponding author. Tel.: +98 342 6226611;

Fax: +98 342 6226617

E-mail addresses: hosseinamiri2010@gmail.com (H. Amiri); pedro.coelho@tecnico.ulisboa.pt (P.J. Coelho) heat flux and temperature distributions over the object to be heat treated (the so-called design surface). The desired conditions over the design surface depend on the process for which the thermal system is built and the thermal properties of the object, such as thermal conductivity, specific heat density, etc. Such problems usually result in equation sets that are mathematically ill-conditioned. This occurs because two conditions are specified on some parts of the system being designed (the design portion), and the unknown conditions on other parts of the system are to be determined. These types of problem are called inverse problems, and regularization [1-3] and optimization [4-12] methods are used to solve them.

In an optimization method, an objective function is defined in such a way that its minimum corresponds to the optimal design outcome. The optimization techniques can be classified as gradient-based methods [4- 
6] and heuristic methods [7-14]. The gradient-based methods are most often used if objective functions are continuously differentiable functions with few local extrema. They work by minimizing the objective function using the local topography of the objective function. The heuristic methods consist of techniques that are based on random sampling of the objective function over the entire feasible region; they can be used for differentiable or non-differentiable objective functions. When design variables are discrete or combinatorial, heuristic methods are the only methods that can be used. Genetic Algorithms (GAs) [711] are the most popular heuristic methods and have gained a permanent position as an optimization algorithm for solving many difficult optimization problems.

Heuristic search methods have been applied to a number of radiation design problems. Li and Yang [9] applied a genetic algorithm to solve the inverse problem for simultaneously determining the single scattering albedo, optical thickness and the phase function from the knowledge of the exit radiation intensities. Safavinejad et al. [10] used a Micro Genetic Algorithm for determining the optimal heater settings in irregular 2-D transparent media. Kim et al. [11,12] used a hybrid genetic algorithm for estimating wall emissivities in a two-dimensional irregular geometry and also applied it to inverse surface radiation analysis in an axisymmetric cylindrical enclosure. Gosselin et al. [13] reviewed the utilization of genetic algorithms in heat transfer problems.

In most of the previous work on the inverse design of radiant enclosures, the number and location of heaters is assumed to be constant and the heater setting that produces the desired condition over the design surface is determined. However, the number and location of heaters greatly affects the efficiency and uniformity with which the materials are heated. Furthermore, the desired conditions on the design surface may be satisfied by using a set of heaters with variable power over the heater surface, but, for practical reasons, heaters with equal power may be preferred in real problems. Porter et al. [14] applied simulated annealing and Tabu search for optimization of a discrete array of radiant heaters in fire simulation equipment. They used an optimization technique to find the optimal location of heaters in a transparent medium. The power of the radiant heaters was fixed in advance. Safavinejad et al. [7] found the number and location of heaters in 2D enclosures containing a transparent medium using a Micro Genetic Algorithm (MGA). The power of the heaters was not fixed in advance, but obtained according to the conservation of energy. Amiri et al. [8] extended the Safavinejad et al. [7] work to participating media, where radiative transfer is the dominant mode of heat transfer. Brittes and Franca [15] considered the inverse boundary design of a $3 \mathrm{D}$ radiative enclosure with a transparent medium. The goal was to determine the power and location of the pre-specified number of heaters that produce uniform temperature and heat flux on the design surface. In order to solve the inverse problem, they proposed a hybrid solution that combines regularization and optimization approaches.

During the operation of a thermal system, the radiative properties of the participating medium and wall properties, such as extinction coefficient, scattering albedo and phase function, and the emissivity of the design surface may change. It is interesting to consider how these changes affect the optimum solution. In this study, calculations have been carried out to investigate the effect of these thermophysical properties on the optimal number and location of heaters over the heater surface that produces the desired condition over the design surface. We concentrate on the design problem in high temperature systems with an absorbing, emitting and scattering medium where radiative transfer is the dominant mode of heat transfer. Here, we extend our previous work [8] to address the effect of thermophysical properties on the optimal solution of inverse problems in radiant enclosures. Two-dimensional irregular enclosures are considered. The inverse problem is formulated as an optimization problem. The Micro Genetic Algorithm is used as an optimization tool for searching for the appropriate number and location of heaters that produce the desired heat flux and temperature distribution over the design surface. First, the accuracy of the direct and inverse solutions is verified, and then the performance of the inverse method is examined by some numerical examples.

\section{Formulation}

\subsection{Direct problem}

The Radiative Transfer Equation (RTE) for an absorbing, emitting and scattering gray medium can be written as [16]:

$$
\begin{aligned}
(\vec{s} . \nabla) I(\vec{r}, \vec{s})=\beta\left\{-I(\vec{r}, \vec{s})+(1-\omega) I_{b}(\vec{r})\right. \\
\left.+\frac{\omega}{4 \pi} \int_{4 \pi} I\left(\vec{r}^{\prime} \vec{s}^{\prime}\right) \varphi\left(\vec{s}^{\prime} \rightarrow \vec{s}\right) d \Omega^{\prime}\right\},
\end{aligned}
$$

in which $\beta=k_{a}+\sigma_{s}$ is the extinction coefficient, $\sigma_{s}$ is the scattering coefficient, and $k_{a}$ is the absorption coefficient; $\omega=\sigma_{s} / \beta$ is the scattering albedo $I_{b}(\vec{r})=\frac{\sigma(T(\vec{r}))^{4}}{\pi}$ is the blackbody radiation intensity at position $\vec{r}$ in the medium; and $I(\vec{r}, \vec{s})$ is the radiation 
intensity at position $\vec{r}$ and in direction $\vec{s} \cdot \varphi\left(\vec{s}^{\prime} \rightarrow \vec{s}\right)$ is the scattering phase function, which denotes the probability that a ray from direction $\vec{s}^{\prime}$ and confined within solid angle $d \Omega^{\prime}$ is scattered into the direction of radiation propagation, $\vec{s}$, confined within a solid angle, $d \Omega$. In this paper, linear anisotropic scattering is considered with the phase function given by:

$$
\varphi\left(\vec{s}^{\prime} \rightarrow \vec{s}\right)=1.0+a_{0}\left(\vec{s}^{\prime} \cdot \vec{s}\right),
$$

where $-1 \leq a_{0} \leq 1$ is an asymmetry factor. Values of the parameter $a_{0}$ are $+1,0$ or -1 for forward, isotropic or backward scattering, respectively.

The boundary conditions for diffusely emitting and reflecting gray walls are defined as:

$$
\begin{aligned}
& I\left(\vec{r}_{w}, \vec{s}\right)=\varepsilon_{w} I_{b}\left(\vec{r}_{w}\right) \\
& \quad+\frac{\left(1-\varepsilon_{w}\right)}{\pi} \int_{n_{w} . \vec{s}^{\prime}<0} I\left(\vec{r}_{w}, \vec{s}^{\prime}\right)\left|\vec{n}_{w} . \vec{s}^{\prime}\right| d \Omega^{\prime}, \quad \vec{n}_{w} . \vec{s}>0
\end{aligned}
$$

where $\varepsilon_{w}$ is the wall emissivity, $I_{b}\left(\vec{r}_{w}\right)$ is the blackbody radiation intensity at the temperature of the boundary surface, and $\vec{n}_{w}$ is the outward unit vector normal to the surface. The radiation intensity depends on the temperature field, and, therefore, the temperature field must be known in order to solve the RTE. Constant temperature and radiative equilibrium cases are considered here. In the case of radiative equilibrium, the temperature field is obtained:

$$
\nabla \cdot \vec{q}=k_{a}\left(4 \pi I_{b}(\vec{r})-\int_{4 \pi} I(\vec{r}, \vec{s}) d \Omega\right)=0,
$$

where $\nabla \cdot \vec{q}$ denotes divergence of the radiative heat flux vector.

Among the different numerical methods for solving RTE, the Discrete Ordinates Method (DOM) has received significant attention and development due to its good accuracy, flexibility and moderate computational requirements. DOM was originally formulated by Chandrasekhar [17] and developed by Lathrop and Carlson [18] and Lathrop [19].

Aiming at reducing ray effects, the Modified Discrete Ordinates Method (MDOM) was developed by Ramankutty and Crosbie [20,21]. Amiri et al. [22,23] used a modified discrete ordinates method to solve radiation [22], and combined conduction-radiation [23] heat transfer problems in 2D irregular geometries. Kim and Kim [24] proposed a hybrid spatial differencing scheme for the discrete ordinates method which reduces false scattering. They showed that this scheme can predict more stable and less smeared results than others. Coelho [25] surveys recent advances on the discrete ordinates method.

In the DOM, the radiative transfer equation is replaced by a discrete set of $M$ coupled differential equations for a finite number of directions, $\vec{s}^{m}(m=$ $1,2, \cdots, M)$. Integrals over solid angles are replaced by a quadrature of order $M$, which yields:

$$
\begin{array}{r}
\left(\vec{s}^{m} \cdot \nabla\right) I\left(\vec{r}, \vec{s}^{m}\right)=-\beta I\left(\vec{r}, \vec{s}^{m}\right)+k_{a} I_{b}(\vec{r}) \\
+\frac{\sigma_{s}}{4 \pi} \sum_{n=1}^{M} I\left(\vec{r}, \vec{s}^{n}\right) \varphi\left(\vec{s}^{n} \rightarrow \vec{s}^{m}\right) w^{n},
\end{array}
$$

in which $w^{n}$ is the quadrature weight of direction $\vec{s}^{n}$. The boundary condition, expressed by Eq. (3), is discretized as:

$$
\begin{aligned}
& I\left(\vec{r}_{w}, \vec{s}^{m}\right)=\varepsilon_{w} I_{b}\left(\vec{r}_{w}\right) \\
& \quad+\frac{\left(1-\varepsilon_{w}\right)}{\pi} \sum_{n=1, \vec{n}_{w} \cdot \vec{s}^{n}<0}^{M} I\left(\vec{r}_{w}, \vec{s}^{n}\right)\left|\vec{n}_{w} . \vec{s}^{n}\right| w^{n}, \\
& \vec{n}_{w} . \vec{s}^{m}>0,
\end{aligned}
$$

and the divergence of radiative heat flux is expressed as:

$$
\nabla \cdot \vec{q}=k_{a}\left(4 \pi I_{b}(\vec{r})-\sum_{n=1}^{M} I(\vec{r}, \vec{s}) w^{n}\right) .
$$

Spatial discretization of the discrete ordinate equations is carried out using the finite volume approach. For a two-dimensional Cartesian coordinate system and for direction $\vec{s}^{m}$ with direction cosines $\mu^{m}$ and $\xi^{m}$, integrating from Eq. (5) yields the discretized equation:

$$
\begin{aligned}
& \left|\mu^{m}\right| A_{x}\left(I_{x, \text { out }}^{m}-I_{x, \text { in }}^{m}\right)+\left|\xi^{m}\right| A_{y}\left(I_{y, \text { out }}^{m}-I_{y, \text { in }}^{m}\right) \\
& =\left(-\beta I_{i, j}^{m}+S_{i, j}^{m}\right) V
\end{aligned}
$$

where:

$$
S_{i, j}^{m}=k_{a} I_{b i, j}+\frac{\sigma_{s}}{4 \pi} \sum_{n=1}^{M} I_{i, j}^{n} \varphi\left(\vec{s}^{n} \rightarrow \vec{s}^{m}\right) w^{n} .
$$

In the above equations, $A_{x}$ and $A_{y}$ are the areas of the control-volume faces normal to $x$ - and $y$-directions, respectively; $V$ is the volume; $I_{i, j}^{m}$ is the intensity at grid node $i, j$ and in direction $\vec{s}^{m}$. The subscripts of the cell face intensities represent the direction $(x$ or $y$ ) and the upstream (in) or downstream (out) face. In this study, the hybrid spatial differencing scheme proposed by Kim and Kim [24] is used to relate the unknown radiation intensities at the cell faces to radiation intensities at neighboring grid nodes. Eqs. (7) and (8) are coupled through term $I_{i, j}^{m}$, and must be solved iteratively to yield radiation intensity and temperature fields. These equations are solved using the procedure described in [26]. 


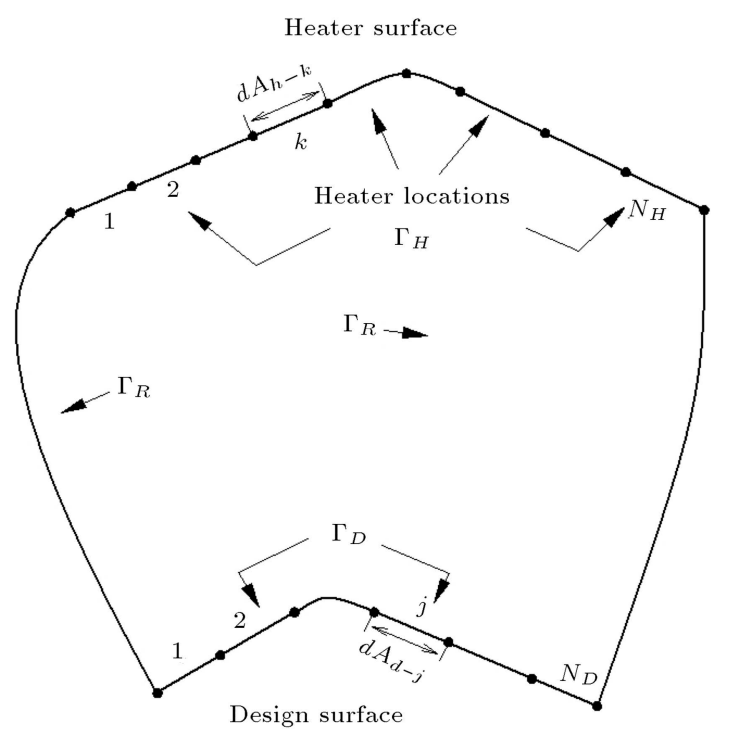

Figure 1. Irregular enclosure, heater and design surfaces.

\subsection{Optimization}

Radiative heat transfer in an irregular enclosure, as shown in Figure 1, is considered. The enclosure contains a gray participating, absorbing, emitting and linear anisotropic scattering medium with uniform properties in radiative equilibrium surrounded by gray and diffusive walls. The boundary conditions are specified over each part of the boundary surface, except on the part corresponding to the heater surface $\left(\Gamma_{H}\right)$. In addition, on the design surface $\left(\Gamma_{D}\right)$, both temperature and heat flux distributions are specified a priori. In the present study, all surfaces, except heater and design surfaces $\left(\Gamma_{R}\right)$, are assumed to be refractory and adiabatic. In order to solve the optimization problem, the heater and the design surfaces are discretized into $N_{H}$ and $N_{D}$ elements, respectively. The desired heat fluxes over the design surface are represented by vector, $\vec{q}_{d}=\left[q_{d-1}, q_{d-2}, q_{d-3}, \cdots, q_{d-j}, \cdots, q_{d-N_{D}}\right]^{T}$. In the optimization processes, the temperature distribution over the design surface is set to the desired temperature, and heat flux distribution over the design surface is used to define the objective function.

Consider $N_{H}$ possible heater locations over the heater surface; there are $2^{N_{H}}$ possible heater configurations over the heater surface. The goal of the optimization process is to find the number $\left(N_{h}\right)$ and location of the equally powered heaters in such a way that they produce the desired heat flux and temperature distributions over the design surface. Since all enclosure walls are adiabatic, except the design and heater surfaces, and the medium is in radiative equilibrium, the energy supplied by the heaters must be equal to the energy absorbed by the design surface. For $N_{h}$ heaters and for the case of equally powered heaters, the heat flux of the heaters can be obtained from the following equation:

$$
q_{h}=\left\{\sum_{j=1}^{N_{D}}\left(\left|q_{d-j}\right| d A_{d-j}\right)\right\} /\left\{\sum_{k=1}^{N_{h}} d A_{h-k}\right\},
$$

where $d A_{d-j}$ is the area of element $j$ on the design surface, $d A_{h-k}$ is the area of element $k$ on the heater surface, $\left|q_{d-j}\right|$ is the absolute value of the desired heat flux on element $j$ of the design surface, $N_{D}$ is the number of discrete elements on the design surface, and $q_{h}$ is the heat flux of the heaters.

For a particular configuration of heaters, all the boundary conditions are known, and the DOM can be used to calculate estimated heat flux distribution, $\vec{q}_{e}=\left[q_{e-1}, q_{e-2}, q_{e-3}, \cdots, q_{e-j}, \cdots, q_{e-N_{D}}\right]^{T}$, over the design surface. Now, an objective function is defined using the heat flux evaluated at $N_{D}$ discrete locations over the design surface:

$$
F(\Psi)=\sum_{j=1}^{N_{D}}\left(q_{d-j}-q_{e-j}(\Psi)\right)^{2},
$$

where $q_{d, j}$ and $q_{e, j}$ are the desired and estimated heat fluxes for element $j$ over the design surface, respectively. The objective function quantifies how close a particular configuration of heaters is to the optimal configuration, in such a way that the minimum of $F(\Psi)$ corresponds to the optimal configuration. The objective function depends on a set of variables contained in $\Psi$, called design parameters, which specify the number, $N_{h}$, and locations of the heaters.

The optimization method is used to modify the initial configuration towards the optimal configuration, iteratively. The solution of the optimization problem is based on the minimization of the objective function, with respect to the unknown parameters, $\Psi$. The micro-genetic algorithm is used for this optimization process, which will be described in the following section.

\subsection{Micro genetic algorithm}

Genetic Algorithm (GA) is a robust parameter search technique based on the concept of natural selection. In the basic GA or Simple GA (SGA), individuals randomly generated at the first stage reach the global optimum through successive iterations, called generations. Each individual in the population is a potential solution to the problem under consideration. During each generation, each individual in the population is evaluated using some measure of fitness (the value of the objective function). After evaluating the fitness of each individual, fitter individuals are selected for reproducing offspring for the next generation. Some of the selected individuals are chosen to find mates and undergo the crossover operation. Then, in order to keep diversity in the population, some of offspring are chosen for mutation operation. The new individuals and some of the best individuals from the last 
generation constitute the new generation. After some number of generations, the algorithm converges to the best string, which, hopefully, represents the optimal or approximate optimal solution to the optimization problem.

Typically, if the population size of SGA is too small, then it might converge to a suboptimal solution. On the other hand, when the population size is too large (for problems with a large number of parameters), the SGA takes longer to converge upon a solution. The SGA cannot work with a small population, as there is not enough diversity in the population pool to allow the SGA to escape from the local optimum. Therefore, different methods are developed to reduce the computational time and efficiency of SGA. One such method, known as the micro-genetic algorithm, developed by Krishnakumar [27], reduces computational time considerably.

In general, Micro-Genetic Algorithm (MGA) can work with populations as small as 5 to 10 individuals. Moreover, MGAs use elitism and convergence checking with re-initialization to obtain optimal or near optimal solutions. The SGA is used in the normal fashion on this small population until the binary strings of each individual differs from that of the best individual by less than a prescribe percentage (here, 5\%). At this point, a new random population is chosen, while keeping the best individual from the previously converged generations, and the evolution process restarts. The population restart strategy avoids premature convergence to local extremes and incorporates diversity in the population. A flowchart and more details concerning MGA are given in $[7,8]$.

In this study, initially, a set of 5 individuals (population of size 5) is randomly generated for the first generation. The length of each string (or an individual in the population) is fixed to $N_{H}$ bits, because there are $N_{H}$ possible heater locations on the heater surface. In each string, "1" signifies that in a given surface location, a heater is installed (heater is on) and " 0 " indicates that the given surface location is an adiabatic surface (heater is off). Uniform crossover is used here, and the probability of crossover, $P_{c}$, has been taken as 0.5 . As the number of generations increases, the genetic algorithm produces better solutions until no improvement is observed. Results from the genetic algorithm depend somehow on the randomly generated numbers (through the seed number in the random generating subroutine), and, thus, they may probably not be the same every time the program runs. Therefore, the search should be performed with different seed numbers before reaching a conclusion as to whether the solution is optimal. In order to be sure that the optimal solution is independent of the seed number, the micro genetic algorithm first runs with three different seed numbers. Then, the optimization code is run with 3 new seed numbers and the lowest objective function in the first set of runs is taken as the initial population. The optimal solution, which is presented in this paper, is the solution with the lowest objective function in the second set of runs.

\section{Validation}

To check the performance and accuracy of the present method in solving direct and inverse radiation problems, two comparisons are carried out. Throughout the paper, heat fluxes and temperatures are nondimensionalized by $Q=q /\left(\sigma T_{\text {ref }}^{4}\right)$ and $T^{*}=T / T_{\text {ref }}$, respectively, in which $T_{\text {ref }}$ is a reference temperature.

\subsection{Direct method}

Consider the radiative heat transfer in a square enclosure with black walls. The medium is assumed to scatter radiation isotropically and neither absorbs nor emits radiant energy $(\omega=1)$. The bottom boundary of the enclosure is at some finite temperature, $T_{b}$ and the other three boundaries are cold (at zero temperature). The calculations were performed using uniform grids with $11 \times 11$ and $21 \times 21$ control volumes, and $S_{4}, S_{6}$ and $S_{8}$ quadratures. A comparison of the dimensionless heat flux distribution over the hot wall and the zonal method results of [28] is shown in Figure 2. The comparison shows that the present results are in good agreement with the zonal solution. It is observed that further refinement of mesh size over $11 \times 11$ and further refinement of quadrature over $S_{6}$ do not change the results considerably. Other validations for the present DOM is given in [24].

\subsection{Inverse method}

Consider radiative heat transfer in the enclosure shown in Figure 3. The enclosure contains an absorbing,

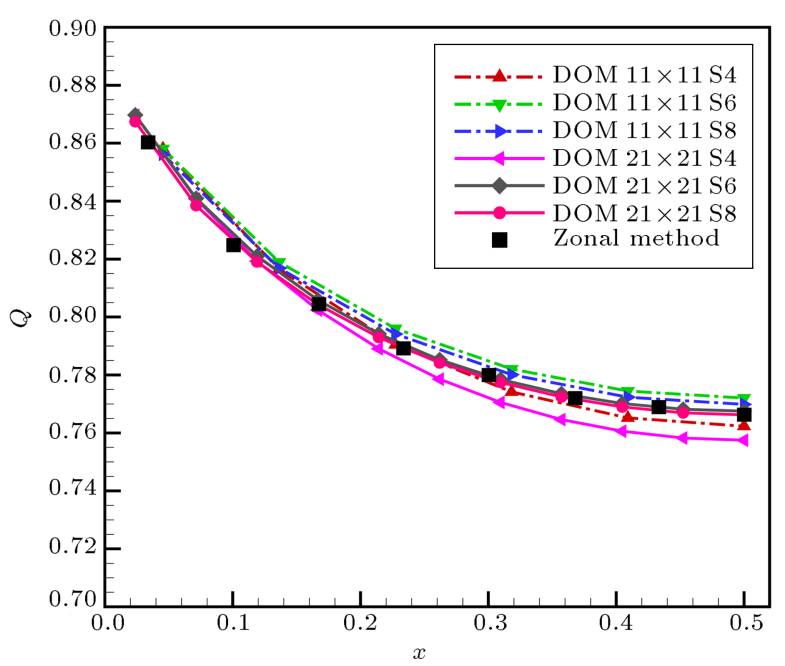

Figure 2. Comparison of dimensionless heat flux over hot wall predicted by the DOM, compared with the results of the zonal method for a square enclosure with black walls. 


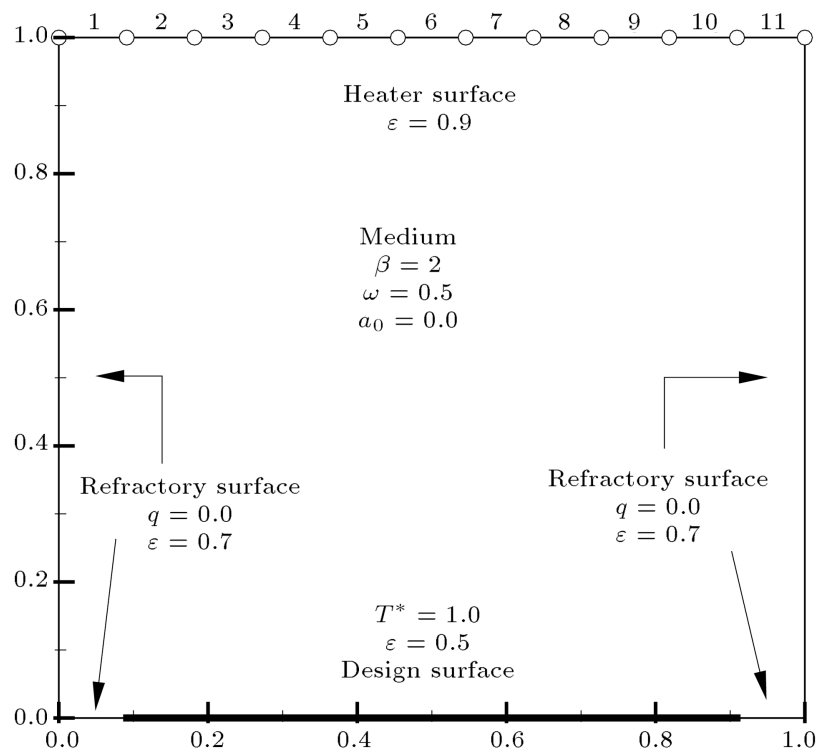

Figure 3. Geometry, boundary conditions and possible heater locations for the rectangular enclosure.

emitting and isotropic scattering medium with $\beta=$ $2 \mathrm{~m}^{-1}, \omega=0.5$ and $a_{0}=0$. The boundary conditions, wall properties and possible heater locations are shown in the figure. To show the ability of the optimization method, three heaters with dimensionless heat flux equal to $Q=6.0$ are placed in locations 1,2 and 3 , and the other locations are assumed to be adiabatic. Then, the direct problem is solved with $11 \times 11$ uniform control volumes and the heat flux over the design surface is calculated. Finally, the calculated heat flux over the design surface is taken as the desired heat flux and the optimization method is applied to find the number and location of the heaters. The extinction coefficient, scattering albedo, wall properties and desired temperature are the same in the direct and inverse problems.

Figure 4 shows the convergence rate of the objective function versus the number of generations for different seed numbers. It shows that the optimization method rapidly finds the exact location of the heaters by searching only a small fraction $\left((12 \times 5) / 2^{11} \times 100=\right.$ $2.93 \%)$ of all possible configurations.

\section{Results and discussion}

\subsection{Example problem}

The optimization methodology is demonstrated by applying it to solve a design problem involving a 2$\mathrm{D}$ diffuse-walled enclosure, shown in Figure 5. The enclosure is filled with a participating medium with an extinction coefficient, scattering albedo, asymmetry factor and the emissivity of a design surface equal to $\beta=2 \mathrm{~m}^{-1}, \omega=0.1, a_{0}=-1$ and $\varepsilon_{D}=0.5$, respectively. The goal of this design problem is to find the optimal number and location of equally powered

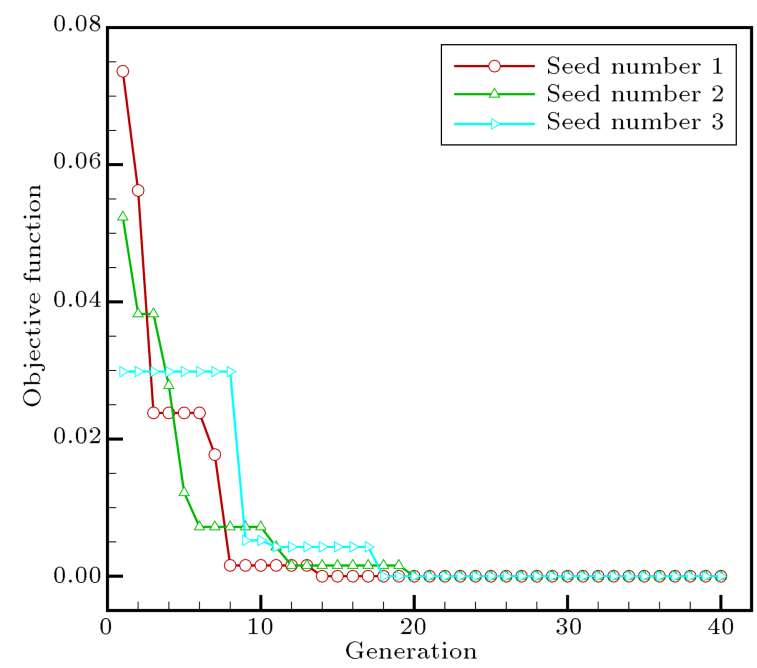

Figure 4. Variation of the fitness function various generations for different seed numbers.

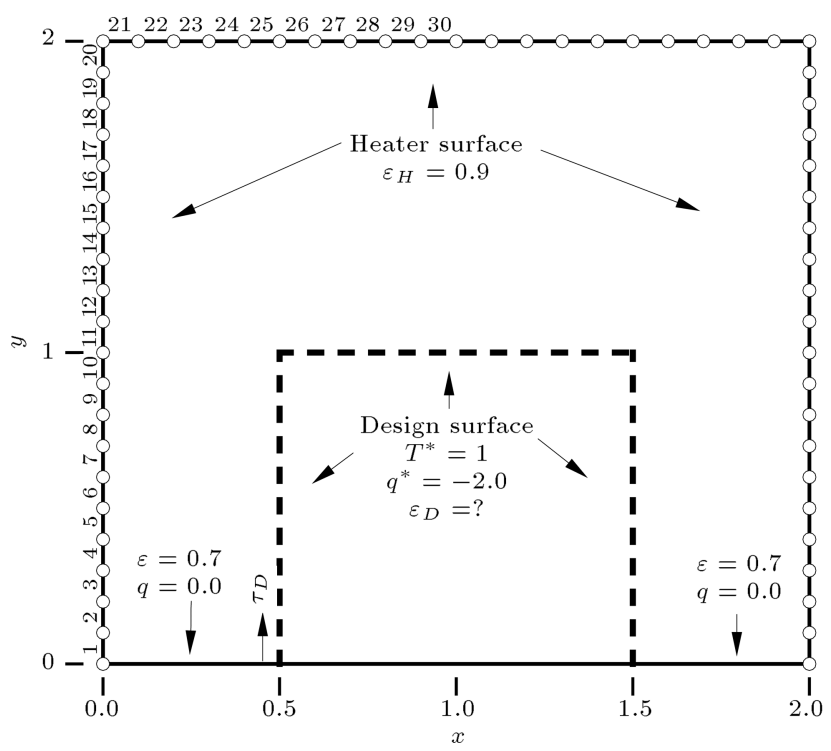

Figure 5. Geometry, boundary conditions, heater locations and heater number of the example problem.

heaters to produce the uniform dimensionless heat flux of $Q_{D}=-2$ and temperature of $T_{D}^{*}=1.0$ over the design surface. The radiative heat transfer has been solved by discretizing the enclosure into a $20 \times 20$ uniformly spaced spatial mesh and $S_{6}$ angular quadrature scheme. Due to the symmetry of the boundary conditions and the configuration, the optimal number and position of heaters must be found from a set of $N_{H}=30$ possible locations in one side of the symmetry line. The same distribution applies over the other side of the heater surface due to its symmetry. There are $2^{30}$ possible configurations for the number and location of heaters. Figure 6 shows locations and the optimal number of heaters over the heater surface. It shows that $N_{h}=12$ and that the number of heaters over the heater surface, $N$, is equal to $2 \times N_{h}=24$. 


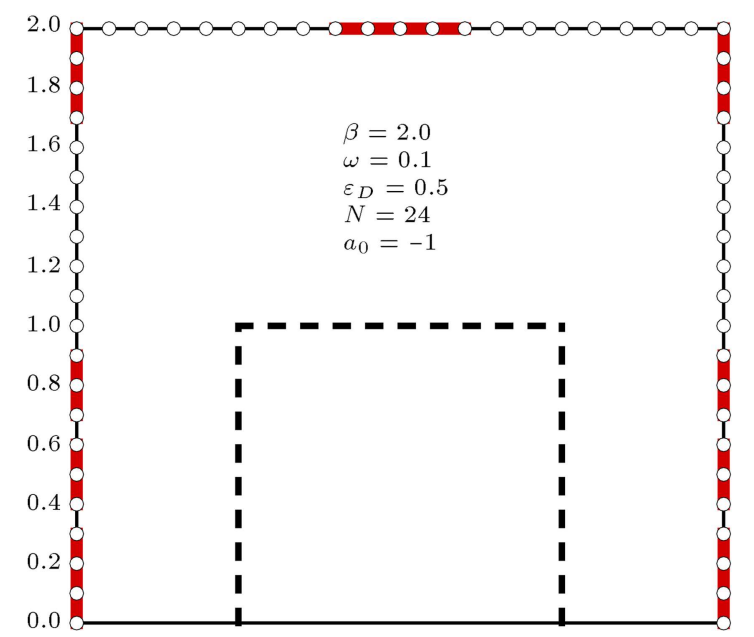

Figure 6. The optimum arrangement of heaters over the heater surface.

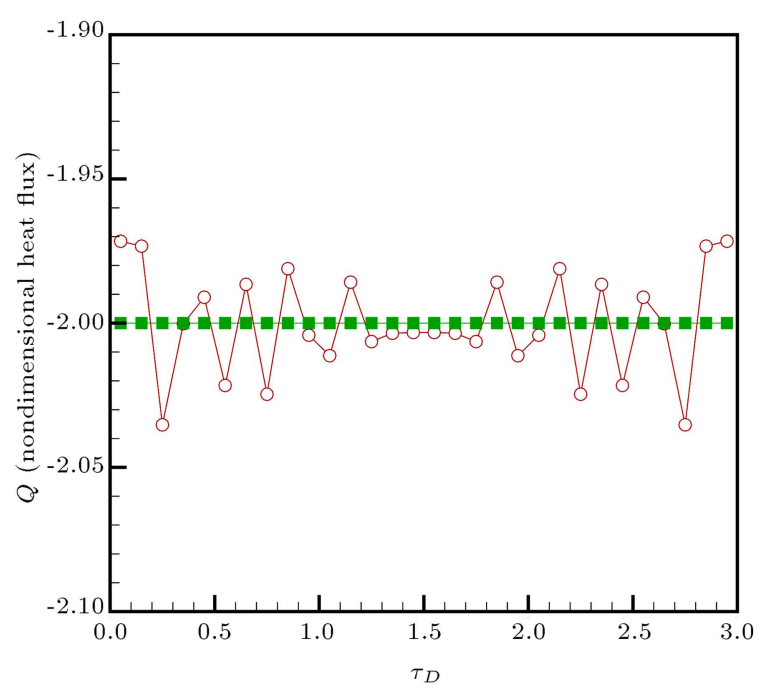

Figure 7. Desired and estimated heat flux over the design surface.

Figure 7 shows the estimated dimensionless heat flux distribution over the design surface. As shown, the uniform desired heat flux distribution is well recovered using the optimization method $(F=0.00973)$. The maximum relative error over the design surface of the enclosure is less than $2 \%$, which is quite acceptable. Figure 8 shows the rate of convergence of the objective function versus the number of iterations for a seed number of three. It shows that the objective function is rapidly converged to a small value by searching only $\left(\left(600 \times 5 / 2^{30}\right) \times 100=2.8 \times 10^{-4} \%\right)$ percent of all possible solutions.

4.1.1. Effect of the number of possible heater locations For practical reasons, the designer may prefer to use larger heaters. For a constant configuration, increasing the area of the heaters $\left(A_{h-k}, k=1,2, \cdots, N_{H}\right)$ decreases the number of possible heater locations, $N_{H}$, and vice versa. In order to see the effect of the number

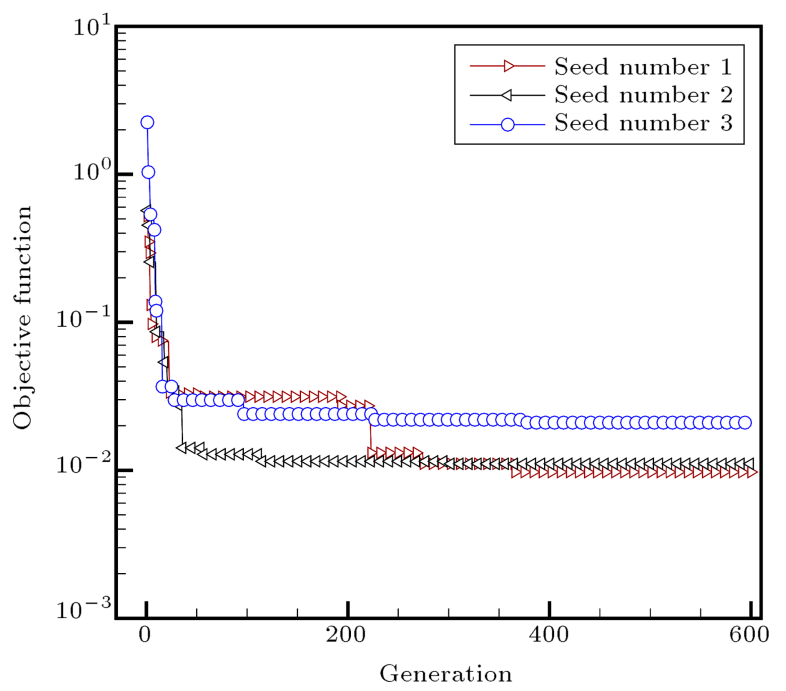

Figure 8. Variation of the fitness function versus generations for different runs.
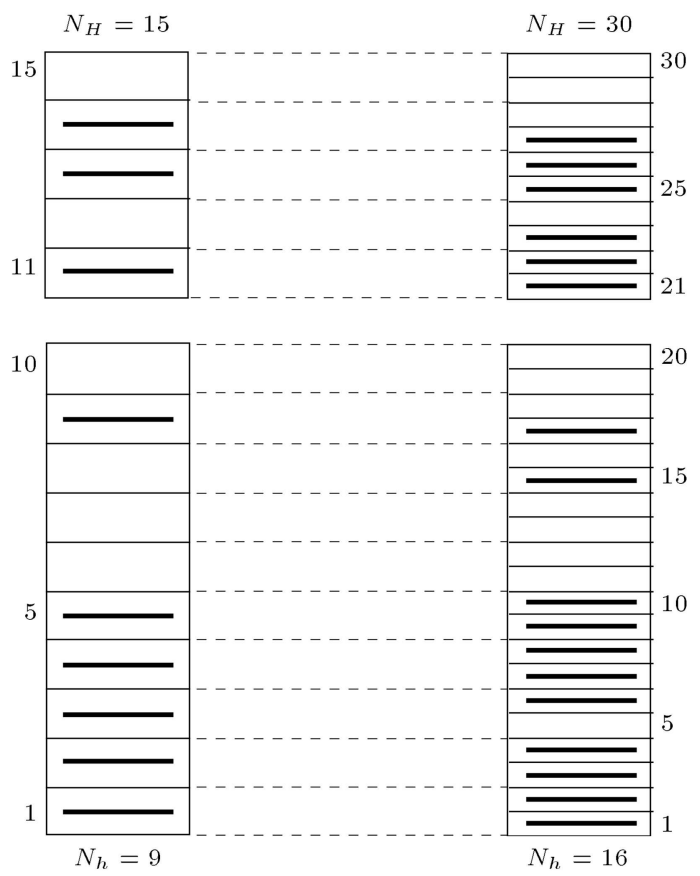

Figure 9. Effect of number of possible heater locations.

of possible heater locations, the optimization process is applied to find an optimal solution for $N_{H}=15$ and $N_{H}=30$. The enclosure, shown in Figure 5, contains an absorbing, emitting and scattering medium, with $\beta=0.1 \mathrm{~m}^{-1}, \omega=0.5$ and $a_{0}=1$. The emissivity of the design surface is $\varepsilon_{D}=0.5$. A uniformly spaced $20 \times 20$ spatial mesh and the $S_{6}$ angular quadrature were used to solve the problem. As Figure 9 shows, the optimal solutions are not exactly the same but they have the same trend. As expected, and shown in Figure 10, the estimated heat flux profile can be made closer to the desired value by using smaller heaters $\left(N_{H}=30\right)$. 


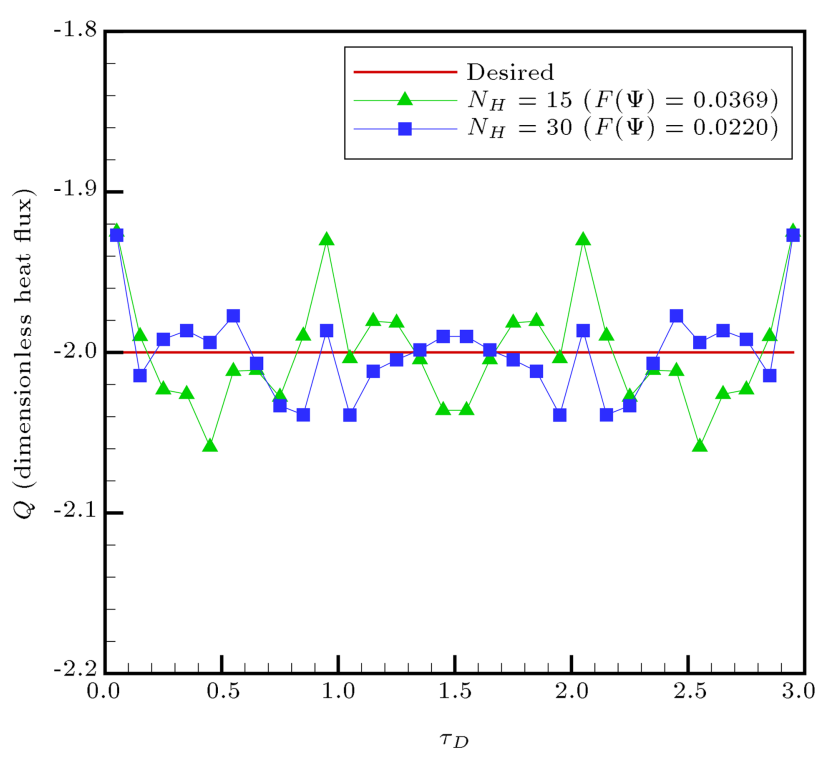

Figure 10. Desired and estimated heat flux over the design surface for two possible heater locations.

\section{Parametric study}

The properties of participating media, such as extinction coefficient, scattering albedo, asymmetry factor, and the emissivity of the design surface, may change. It is interesting to consider how these changes can affect the optimal number and location of the heaters. The following calculations have been carried out to investigate the effect of the above parameters on the optimal number and location of equally powered heaters over the heater surface to produce the uniform dimensionless heat flux of $Q_{D}=-2$ and temperature of $T_{D}^{*}=1.0$ over the design surface in an enclosure, as shown in Figure 6. For all cases, we have used a $20 \times 20$ uniform grid and the $S_{6}$ angular quadrature scheme.

\subsection{Extinction coefficient}

Consider radiative heat transfer in the enclosure shown in Figure 5 with an absorbing, emitting and isotropic scattering medium with $\omega=0.5, a_{0}=0$ and wall emissivity of $\varepsilon_{D}=0.5$. Figure 11 shows the optimal number and location of heaters for various extinction coefficients. Figure 12 shows the estimated and desired heat fluxes over the design surface for some of these extinction coefficients, and Figure 13 shows the convergence rate of the objective function with generations for different extinction coefficients. From the above figures, two effects are clear. First, as the extinction coefficient increases, the number of heaters decreases and, thus, the power of the heaters will increase. Second, the locations of the heaters go towards the regions in front of the design surface. Also, it is seen that optimal solutions are less affected by variations of the extinction coefficient for optically thick media, and the objective function convergences to a relatively higher value for $\beta>5 \mathrm{~m}^{-1}$. The

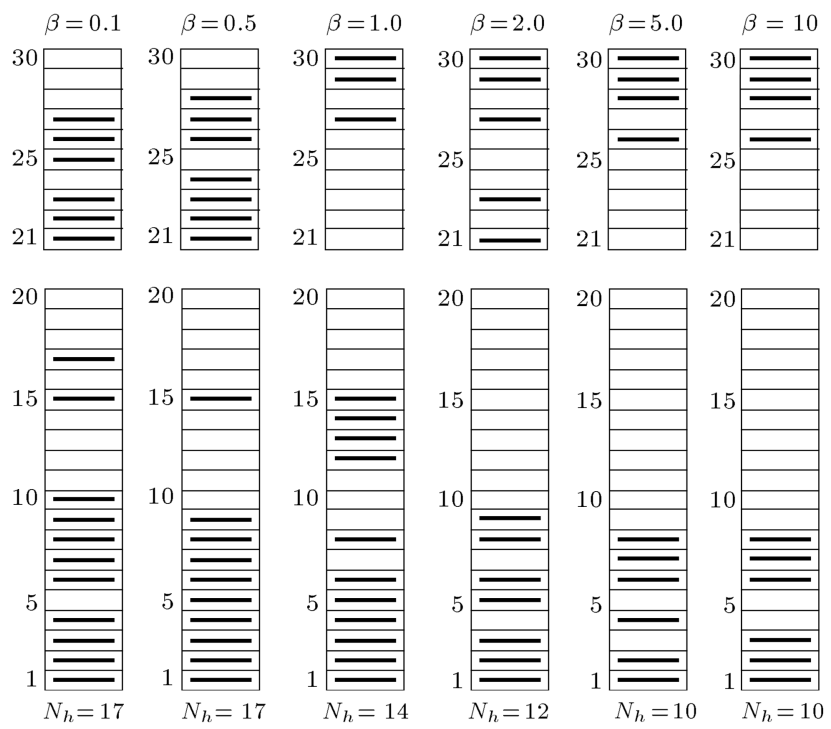

Figure 11. Effect of extinction coefficient on optimal solution for medium with $\omega=0.5, a_{0}=0$ and $\varepsilon_{D}=0.5$.

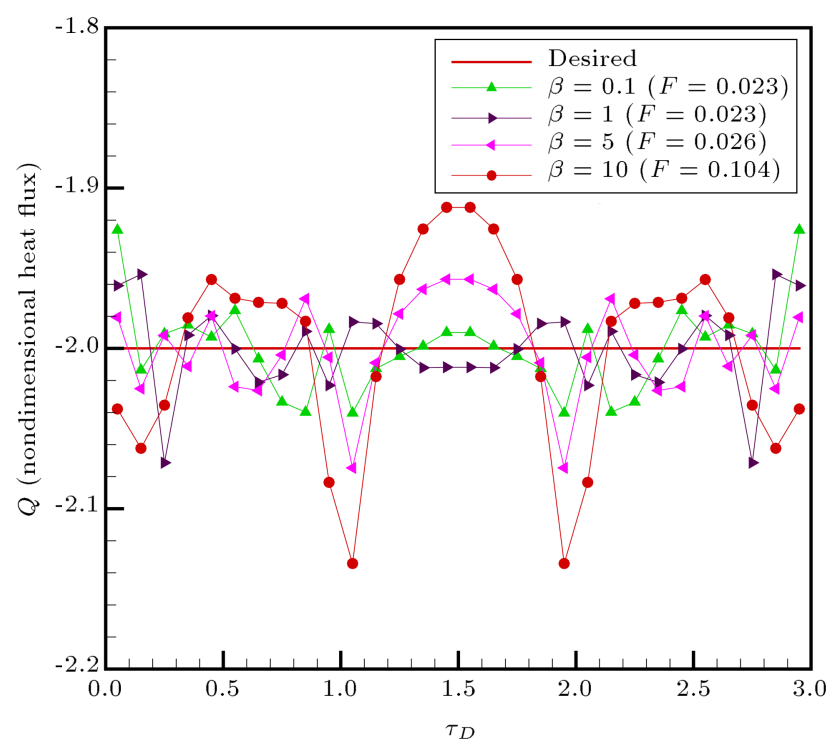

Figure 12. Desired and estimated heat fluxes over the design surface for various extinction coefficients.

reason for this behavior is that, as the extinction coefficient and, therefore, the optical thickness of the medium decreases, the radiation emitted by the heater surface dominates the heat flux on the design surface, whereas the local gas temperature will control the heat flux on the design surface as the extinction coefficient increases.

\subsection{Scattering albedo}

In order to investigate the effect of the scattering albedo, the optimization method has been used to find the number and best location of equally powered heaters in an enclosure with $a_{0}=-1$, and $\varepsilon_{D}=0.5$, and with two extinction coefficients, $\beta=0.5 \mathrm{~m}^{-1}$ and $\beta=2.0 \mathrm{~m}^{-1}$, for various scattering albedos. Optimal 


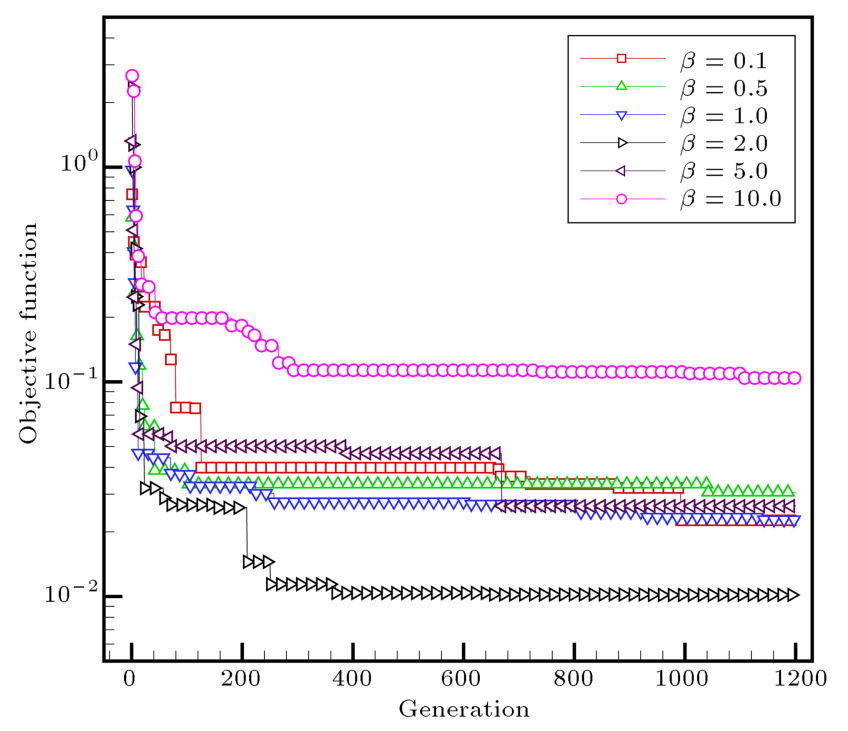

Figure 13. Variation of the objective function versus generations for different extinction coefficients.
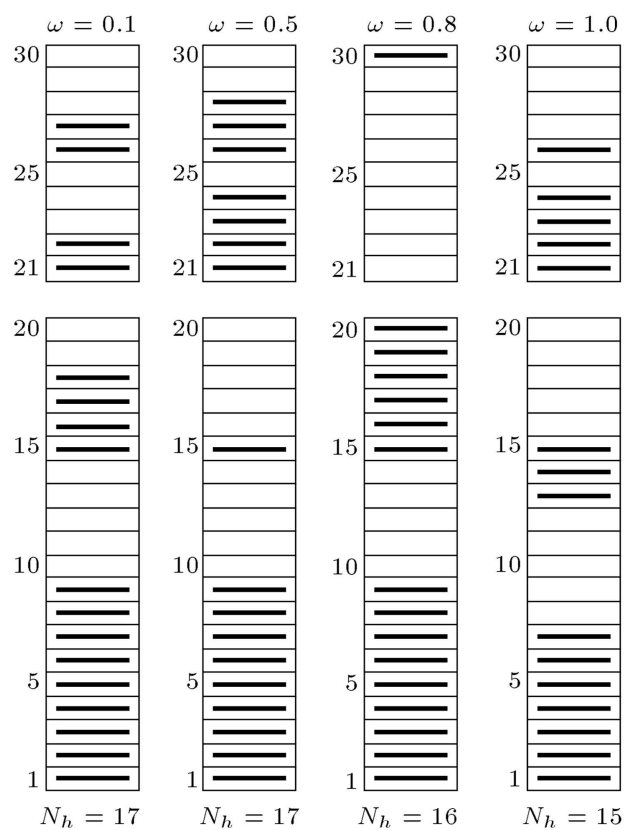

Figure 14. Effect of scattering albedo on optimal solution for $\beta=0.5 \mathrm{~m}^{-1}, a_{0}=-1$ and $\varepsilon_{D}=0.5$.

solutions are shown in Figures 14 and 15. changes As seen from these figures, scattering albedo has a profound effect on the optimal solutions, but a clear cut conclusion cannot be reached as to how the scattering albedo correlates with optimal solutions.

\subsection{Asymmetry factor}

In order to investigate the effect of the asymmetry factor, radiative heat transfer in an enclosure with $\omega=0.5$, and $\varepsilon_{D}=0.5$, and with two extinction coefficients, $\beta=1.0 \mathrm{~m}^{-1}$ and $\beta=5.0 \mathrm{~m}^{-1}$, have been considered for various asymmetry factors, and optimal solutions are shown in Figures 16 and 17. Results

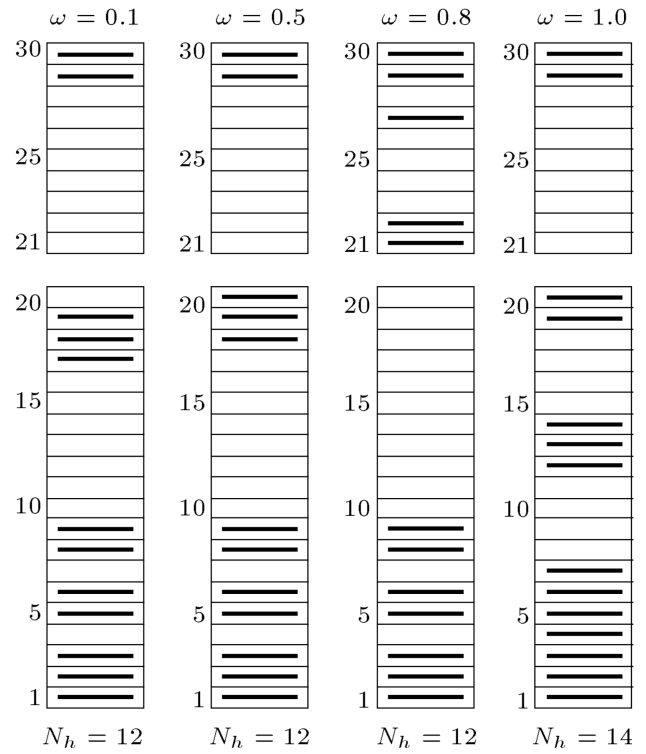

Figure 15. Effect of scattering albedo on optimal solution for $\beta=2.0 \mathrm{~m}^{-1}, a_{0}=-1$ and $\varepsilon_{D}=0.5$.
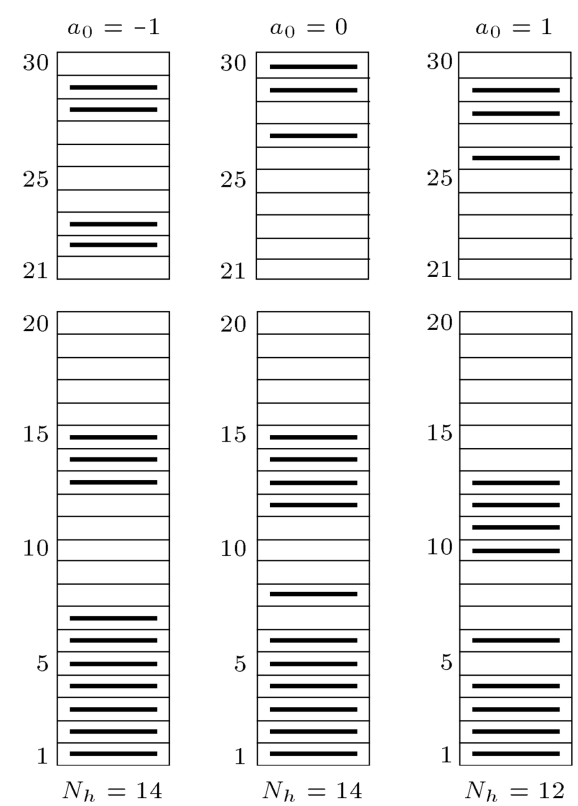

Figure 16. Effect of asymmetry factor on optimal solution for $\beta=1.0 \mathrm{~m}^{-1}, \omega=0.5$ and $\varepsilon_{D}=0.5$.

show that the asymmetry factor variation the optimal solution considerably for $\beta=1.0 \mathrm{~m}^{-1}$, but only slightly changes the optimal solution for $\beta=5.0 \mathrm{~m}^{-1}$.

\subsection{Emissivity of design surface}

The optimal number and location of heaters in a medium with $\omega=0.5$, and $a_{0}=0$, and two absorption coefficients, $\beta=0.1 \mathrm{~m}^{-1}$ and $\beta=5 \mathrm{~m}^{-1}$, have been obtained for various emissivities of design surfaces, and are shown in Figures 18 and 19, respectively. As seen from these figures, variation of the emissivity of the design surface changed the optimal configuration, but this variation is small for high extinction coefficients. 

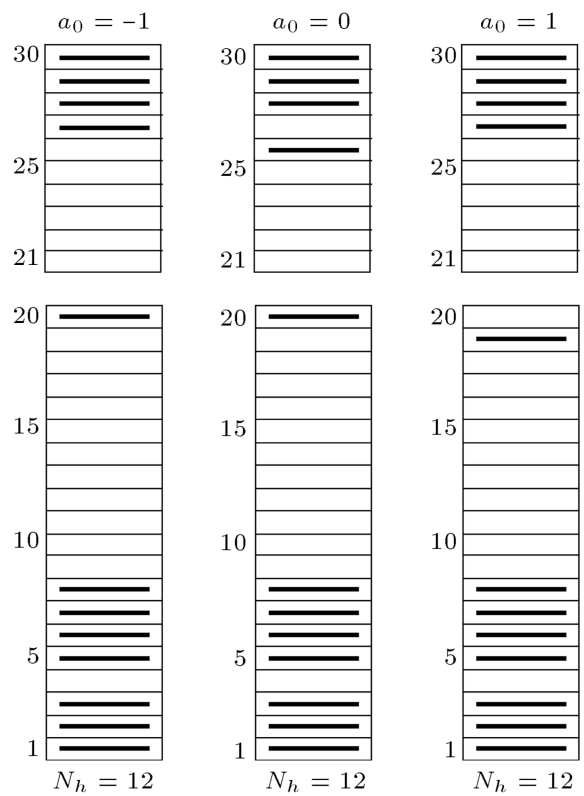

Figure 17. Effect of asymmetry factor on optimal solution for $\beta=5.0 \mathrm{~m}^{-1}, \omega=0.5$ and $\varepsilon_{D}=0.5$.
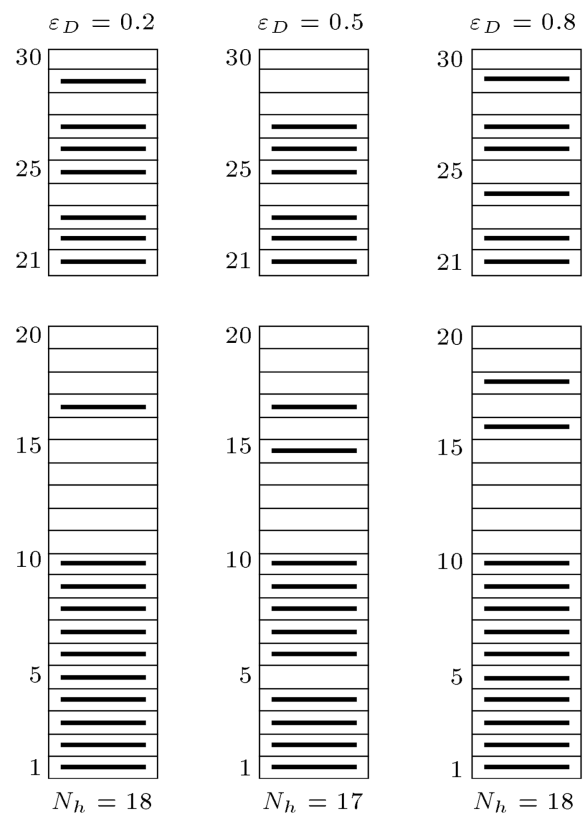

Figure 18. Effect of emissivity of design surface on optimal solution for $\beta=0.1 \mathrm{~m}^{-1}, \omega=0.5$ and $a_{0}=0$.

\section{Conclusion}

In this paper, discrete optimization problems of radiative heat transfer in participating media with irregular geometries have been studied. The genetic algorithm has been used to find the optimum number and location of equally powered heaters to produce a desired temperature and heat flux distribution over the design surface of radiant enclosures. The medium has been assumed to be absorbing, emitting and linear anisotropic scattering. The discrete ordinates method has been
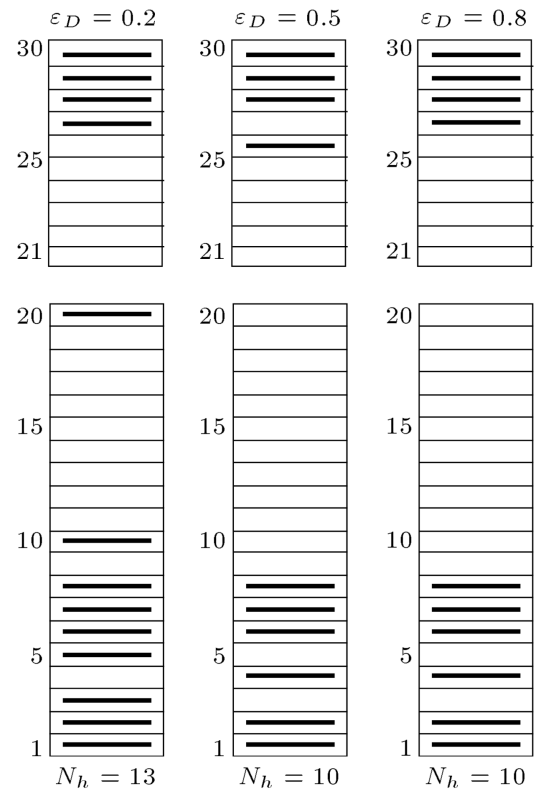

Figure 19. Effect of emissivity of design surface on optimal solution for $\beta=5.0 \mathrm{~m}^{-1}, \omega=0.5$ and $a_{0}=0$.

employed to solve the radiative transfer equation. The micro genetic algorithm has been used to minimize an objective function, expressed as the sum of the square difference between estimated and desired heat fluxes over the design surface. The method is able to solve combinatorial optimization problems for radiative heat transfer in such a way that the temperature and heat flux distributions over the design surface converges to the desired profiles. Results show that the estimated values of heat transfer are in good agreement with the desired values within an acceptable range of errors. The effects of medium and wall characteristics, such as extinction coefficient, scattering albedo, asymmetry factor and emissivity of the design surface, on the optimal solution have been studied in detail. The results indicate that variation in medium and wall properties change the optimal solution considerably for low optical thicknesses (extinction coefficient), but they have little influence on the optimal solution at high optical thicknesses. Therefore, the designer must determine the medium properties more precisely when the extinction coefficient is low. Results also show that even a small change in radiative properties changes the optimal solution. The reason for this behavior is that radiative heat transfer is non-linear and makes the inverse problem more ill-conditioned.

\section{Nomenclature}

$A_{x}, A_{y} \quad$ Areas of the control-volume faces normal to the $x$ and $y$ directions $\left(\mathrm{m}^{2}\right)$

$a_{0} \quad$ Asymmetry factor

$d A \quad$ Element area $\left(\mathrm{m}^{2}\right)$ 
$F(\Psi) \quad$ Objective function

$k_{a}$

$\vec{n}_{w}$

$N_{H}$

$N_{h}$

$N$

$N_{D} \quad$ Number of discrete elements on the design surface

$I_{b}(\vec{r}) \quad$ Black body radiation intensity $\left(\mathrm{Wm}^{-2} \mathrm{Sr}^{-1}\right)$

$I(\vec{r}, \vec{s}) \quad$ Radiation intensity at position $\vec{r}$ and in direction $\vec{s}\left(\mathrm{Wm}^{-2} \mathrm{Sr}^{-1}\right)$

$I_{i, j}^{m} \quad$ Intensity at grid node $i, j\left(\mathrm{Wm}^{-2} \mathrm{sr}^{-1}\right)$

$Q\left(=\frac{q}{\sigma T_{\mathrm{ref}}^{4}}\right)$ Dimensionless heat flux

$\vec{q} \quad$ Radiative heat flux vector $\left(\mathrm{Wm}^{-2}\right)$

$q_{d} \quad$ Desired heat flux $\left(\mathrm{Wm}^{-2}\right)$

$q_{e} \quad$ Estimated heat flux $\left(\mathrm{Wm}^{-2}\right)$

$q_{w} \quad$ Wall heat flux $\left(\mathrm{Wm}^{-2}\right)$

$\vec{r} \quad$ Position vector $(\mathrm{m})$

$\vec{s} \quad$ Geometric path vector $(\mathrm{m})$

$S_{i, j}^{m} \quad$ Source term in Eq. (9)

$T \quad$ Absolute temperature (K)

$T_{\text {ref }} \quad$ Reference temperature (K)

$V \quad$ Volume $\left(\mathrm{m}^{3}\right)$

$w^{j} \quad$ Quadrature weight of the direction $\vec{s}^{j}$

\section{Greek symbols}

$\beta=k_{a}+\sigma_{s} \quad$ Extinction coefficient $\left(\mathrm{m}^{-1}\right)$

$\Gamma_{D} \quad$ Design surface

$\Gamma_{H} \quad$ Heater surface

$\Gamma_{R} \quad$ Refractive surface

$\beta \quad$ Extinction coefficient

$\omega=\sigma_{s} / \beta \quad$ Scattering albedo

$\varepsilon_{w} \quad$ Wall emissivity

$\sigma_{s} \quad$ Scattering coefficient $\left(\mathrm{m}^{-1}\right)$

$\sigma \quad$ Stefan-Boltzmann constant $=$ $5.67 \times 10^{-8} \mathrm{Wm}^{-2} \mathrm{~K}^{-4}$

$\mu^{m}, \xi^{m} \quad x$ and $y$ cosines of $\vec{s}^{m}$ direction

$\gamma_{x}, \gamma_{y} \quad$ Spatial differencing weights to $x$ and $y$ directions

$\varphi\left(\vec{s}^{\prime} \rightarrow \vec{s}\right) \quad$ Scattering phase function

$\Omega \quad$ Solid angle (sr)

$\theta \quad$ Dimensionless temperature

$\Psi \quad$ Design parameters

\section{Subscripts}

in

$d, h \quad$ Design and heater

$R \quad$ Radiation or refractive

out Downstream

$w \quad$ Wall

\section{References}

1. Daun, K.J., Erturk, K. and Howell, J.R. "Inverse design methods for high-temperature systems", The Arabian Journal for Science and Engineering, 27(2C), pp. 3-48 (2002).

2. Daun, K.J. and Howell, J.R. "Inverse design methods for radiative transfer systems", JQSRT, 93, pp. 43-60 (2005).

3. Daun, K.J. and Howell, J.R. "Regularization of inverse boundary design radiative heat transfer problems", JQSRT, 104, pp. 171-195 (2007).

4. Hosseini Sarvari, S.M., Mansouri, S.H. and Howell J.R. "Inverse boundary design radiation problem in absorbing-emitting media with irregular geometry", Numerical Heat Transfer, Part A, 43, pp. 565-584 (2003).

5. Daun, K.J. and Howell, J.R. "Inverse design methods for radiative transfer systems", JQSRT, 93, pp. 43-60 (2005).

6. Moghadassiana, B. and Kowsary, F. "Inverse boundary design problem of natural convection-radiation in a square enclosure", Int. J. Thermal Sciences, 75, pp. 116-126 (2014).

7. Safavinejad, A., Mansouri, S.H., Sakurai, A. and Maruyama, S. "Optimal number and location of heaters in $2 \mathrm{D}$ radiant enclosures composed of specular and diffuse surfaces using micro-genetic algorithm", Applied Thermal Engineering, 29, pp. 10751085 (2009).

8. Amiri, H., Mansouri, S.H., Safavinejad, A. and Coelho, P. "The optimal number and location of discrete radiant heaters in enclosures with the participating media using the micro genetic algorithm", Numerical Heat Transfer, Part A, 60, pp. 461-483 (2011).

9. Li, H.Y. and Yang, C.Y. "A genetic algorithm for inverse radiation problems", Int. J. Heat and Mass Transfer, 40, pp. 1545-1549 (1997).

10. Safavinejad, A., Mansouri, S.H., Sakurai, A. and Maruyama, S. "Optimal boundary design of radiant enclosures using micro-genetic algorithm", J. Thermal Science and Technology, 3(2), pp. 179-194 (2008).

11. Kim, K.W., Baek, S.W., Kim, M.Y. and Ryou, H.S. "Estimation of emissivities in a two-dimensional irregular geometry by inverse radiation analysis using hybrid genetic algorithm", JQSRT, 87, pp. 1-14 (2004). 
12. Kim, K.W. and Baek, S.W. "Inverse surface radiation analysis in an axisymmetric cylinder enclosure using a hybrid genetic algorithm", Numerical Heat Transfer, Part A, 46, pp. 367-381 (2004).

13. Gosselin, L., Tye-Gingras, M. and Mathieu-Potvin, F. "Review of utilization of genetic algorithms in heat transfer problems", Int. J. Heat and Mass Transfer, 52, pp. 2169-2188 (2009).

14. Porter, J.M., Larsen, M.E., Barnes, J.W. and Howell, J.R. "Metaheuristic optimization of a discrete array of radiant heaters", ASME J. Heat Transfer, 128, pp. 1031-1040 (2006).

15. Brittes, R. and França, A. "Hybrid inverse method for the thermal design of radiative heating systems", Int. Heat and Mass Transfer, 57, pp. 48-57 (2013).

16. Modest, M.F., Radiative Heat Transfer, 2nd Ed., Academic Press, New York (2003).

17. Chandrasekhar, S., Radiative Transfer, Clarendon Press, Oxford (1950).

18. Lathrop, K.D. and Carlson, B.G. "Discrete ordinates angular quadrature of the neutron transport equation", Technical Information Series Report LASL3186, Los Alamos Scientific Laboratory (1965).

19. Lathrop, K.D. "Use discrete ordinates methods for solution of photon transport problems", J. Nuclear Science Energy, 24, pp. 381-388 (1966).

20. Ramankutty, M.A. and Crosbie, A.L. "Modified discrete ordinates solution of radiative transfer in twodimensional rectangular enclosures", JQSRT, 57, pp. 107-140 (1997).

21. Ramankutty, M.A. and Crosbie, A.L. "Modified discrete ordinates solution of radiative transfer in threedimensional rectangular enclosures", JQSRT, 60, pp. 103-134 (1998).

22. Amiri, H., Mansouri, S.H. and Coelho, P.J. "Application of the modified discrete ordinates method with the concept of blocked-off region to irregular geometries", Int. J. Thermal Sciences, 50(4), pp. 515-524 (2011).

23. Amiri, H., Mansouri, S.H. and Coelho, P.J. "Application of modified discrete ordinates method to combined conduction-radiation heat transfer problems in irregular geometries", Int. J. Numerical Methods for Heat and Fluid Flow, 22(7), pp. 862-879 (2012).

24. Kim, I.K. and Kim, W.S. "A hybrid spatial differencing scheme for discrete ordinates method in $2 \mathrm{D}$ rectangular enclosures", Int. J. Heat and Mass Transfer, 44, pp. 575-586 (2001).
25. Coelho, P.J. "Advances in the discrete ordinates and finite volume methods for the solution of radiative heat transfer problems in participating media", JQSRT, 145, pp. 121-146 (2014).

26. Amiri, H., Mansouri, S.H. and Safavinejad, A. "Combined conductive and radiative heat transfer in an anisotropic scattering participating medium with irregular geometries", Int. J. Thermal Sciences, 49, pp. 492-503 (2010).

27. Krishnakumar, K. "Micro-genetic algorithms for stationary and non-stationary function optimization", SPIE Intelligent Control and Adaptive Systems, 1196, pp. 289-296 (1989).

28. Rousse, D.R., Gautier, G. and Sacadura, J.F. "Numerical predictions of two dimensional conduction, convection and radiation heat transfer: II. Validation", Int. J. Thermal Sciences, 39, pp. 315-331 (2000).

\section{Biographies}

Hossein Amiri received his BS degree from Birjand University, Iran, in 2003, and MS and PhD degrees from Shahid Bahonar University, Kerman, Iran, in 2005 and 2011, respectively, all in Mechanical Engineering. His main fields of interest and expertise are CFD modeling and numerical simulation of radiative heat transfer and fuel cells. He is currently Professor of Mechanical Engineering in the Department of Energy at the Institute of Science and High Technology and Environmental Sciences in the Graduate University of Advanced Technology, Kerman, Iran.

Pedro Coelho has a BS degree in Mechanical Engineering (1984), an MS degree in Energy Transfer and Conversion (1988), and $\mathrm{PhD}$ degrees in Mechanical Engineering (1992) and Habilitation in Mechanical Engineering (2005). All the degrees were obtained from Instituto Superior Técnico at the Technical University of Lisbon, where he is currently Associate Professor in the area of Thermofluids and Technologies of Energy Conversion in the Mechanical Engineering Department and where he has been teaching since 1984. He has more than 60 papers published in international journals, and more than 90 papers presented at international conferences. His research is in the field of numerical simulation of heat transfer and combustion problems. Specific areas of interest are radiation models, gas radiative properties, turbulenceradiation interaction, turbulent diffusion flames, mild combustion and industrial combustion equipment. 